\title{
Fuzzy System Analysis of Beach Litter Components
}

\author{
Can Elmar Balas \\ Gazi University, Faculty of Engineering and Architecture \\ Civil Engineering Department, 06570 Ankara, Turkey \\ cbalas@gazi.edu.tr
}

\begin{abstract}
Tourist beaches on the southern coast of Turkey are surveyed in order to facilitate a standardised fuzzy approach to be used in litter prediction and to assess the aesthetic state of the coastal environment for monitoring programs. During these surveys the number of litter items on beaches were counted and recorded in different categories. The main source of litter on beaches was determined as "beach users". A fuzzy system was developed to predict the classification of the beaches, since uncertainty was generally inherent in beach work due to the high variability of beach characteristics and the sources of litter categories. This resulted in effective utilization of "the judgment and knowledge of beach users" in the evaluation of beach gradings.
\end{abstract}

\section{Introduction}

Marine litter is defined as the solid materials of human origin that are discarded at sea or reaches the sea through waterways or domestic or industrial outfall [1]. Litter in the marine environment leads to numerous problems adversely affecting coastal development sectors. Prevention at source is one of the most important strategies in enabling the reduction of litter pollution, and for this aim to be achieved strong links between measurement and management need to be realized. Five beaches on the south coast of Turkey are studied with regards to type and amount of litter on each beach. The surveys were carried out at over $100 \mathrm{~m}$ wide transects of the beaches.

Four site investigations were performed, first being on 20-29 October 2000, second on 10-19 November 2000, third on 15-26 March 2001 and the last on 2-13 June 2001. Field litter studies were conducted on some of the most attractive tourist beaches of the Turkish Riviera (Antalya) coast, namely Cirali, Konyaalti, Kemer, Side and Belek. For a $100 \mathrm{~m}$ stretch of beach located on the normal access points, all litter items were enumerated and placed in their respective categories/grades [2]. Litter amounts collected ranged from 18 to 743 -items/100 m stretch of beach. Litter items were graded from the best (Grade A) to worst case (Grade D) as shown in Table 1.

\section{Litter Prediction by Fuzzy System}

Field measurements indicated that the main beach litter item (the most abundant in terms of quantity) was the general litter category. The number of litter items in other categories was low and oil pollution was not observed. Konyaaltı beach was rated to 
be in bad conditions on the first three site surveys. The reasons were its nearness to the city center, the demolished refreshment kiosks due to the implementation of the municipality plan of Antalya and the difficulty in controlling the pollution on its seven-kilometer long beach. However on the last survey, the grading of Konyaalt beach increased from " $\mathrm{D}$ " to "B", due to the improvement in tourism facilities implemented by the municipality of Antalya. This indicated a sharp decline in the amount of litter items on the beach with the beginning of the tourism season.

Table 1. Categories for grading of beaches

\begin{tabular}{|c|c|c|c|c|c|c|}
\hline & Category & Type & $\mathrm{A}$ & B & $\mathrm{C}$ & $\mathrm{D}$ \\
\hline \multirow[t]{2}{*}{1} & Sewage & General & 0 & $1-5$ & $6-14$ & $>15$ \\
\hline & Related Debris & $\begin{array}{c}\text { Cotton } \\
\text { Buds }\end{array}$ & $0-9$ & $10-49$ & $50-99$ & $>100$ \\
\hline 2 & Gross Litter & & 0 & $1-5$ & $6-14$ & $>15$ \\
\hline 3 & General Litter & & $0-49$ & $\begin{array}{l}50- \\
499\end{array}$ & $500-999$ & $>1000$ \\
\hline 4 & Harmful Litter & $\begin{array}{c}\text { Broken } \\
\text { Glass }\end{array}$ & 0 & $1-5$ & $6-24$ & $>25$ \\
\hline 5 & Accumulations & Number & 0 & $1-4$ & $5-9$ & $>10$ \\
\hline 6 & Oil & & Absent & Trace & Noticeable & Objectionable \\
\hline 7 & Faeces & & 0 & $1-5$ & $6-24$ & $>25$ \\
\hline
\end{tabular}

The Moonlight beach and the public beach of Kemer were in good condition with a beach grading of "B". With the beginning of tourism season, these beaches were regularly cleaned. Çıralı and Belek are the selected coastal sites for 'Coastal Management and Tourism Project'. World Wide Fund is conducting the project with World Bank funding. Çıralı beach was in good condition with a beach grading of " $\mathrm{B}$ ". There were less tourism facilities when compared to other beaches studied, hence the grade of the beach did not change with the tourism season. Side beach obtained a low degree of grading in surveys, mostly due to faeces observed. With the beginning of tourism season, the beach was regularly cleaned and the overall grade of Side beach increased to "B". The variation can be attributed to national holidays, stormy wheather conditions, demolition and construction of beach facilities and the tourism season.

A fuzzy system of artificial intelligence [3] was developed in this paper, which had input parameters of general litter and sewage related debris, and an output parameter of the grading of litter categories. In the fuzzification process of the system inputs, which were the number of general litter and sewage related debris items, the grading criteria was utilized. 


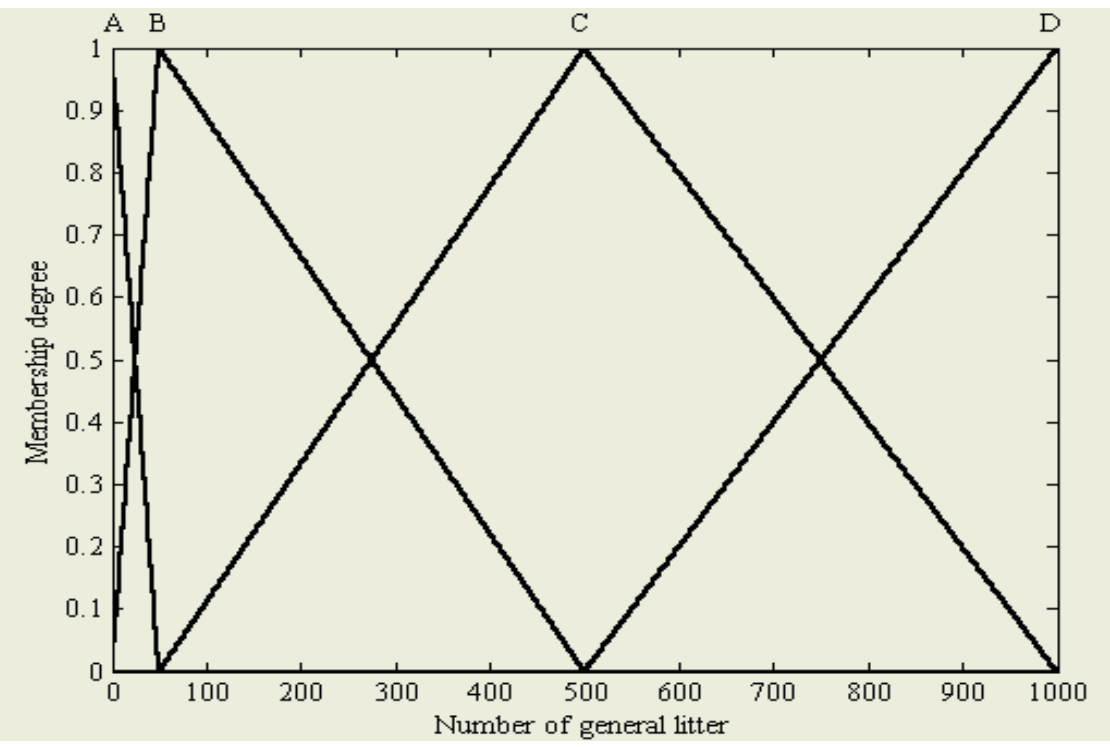

Fig. 1. Fuzzy input sets and the membership functions of the grading system for the category of general litter

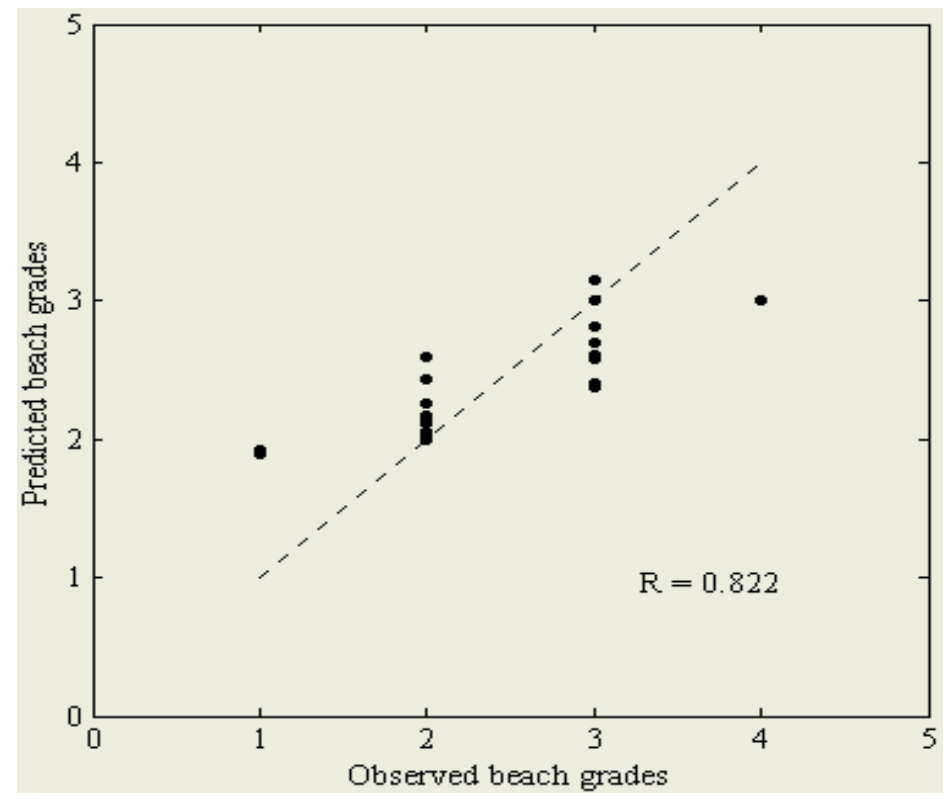

Fig. 2. Comparison of beach grades of the field study with the predicted grades of fuzzy system

The fuzzy input sets and membership functions of these variables were obtained for the grading of A, B, C, D, from the best to worst case depending on the number of 
litter items measured on beaches (Figure 1). Fuzzy output sets for the grading of litter categories (A: excellent, B: good, C: average and D: worst) were coded as 1,2, 3 and 4 , respectively. The fuzzy rule based system was established by the Cartesian product (product space) of fuzzy input sets, which resulted the in the logical base of 16 fuzzy rules. The fuzzy sets of grades were slightly modified to assess the uncertainties inherent in other litter categories by including supplementary adjectives of "very good $\left(\mathrm{B}^{+}\right)$, to some extend good $\left(\mathrm{B}^{-}\right)$, above average $\left(\mathrm{C}^{+}\right)$, below average $\left(\mathrm{C}^{-}\right)$and bad $\left(\mathrm{D}^{+}\right)$", i.e. the maximum number of litter items, greater than a certain limiting value in related categories will decrease a half grade in the input fuzzy subset of rules. Therefore, potentially harmful litter, gross litter and accumulations of litter exceeding their limits given in Table 1, will decrease the grading definition in fuzzy rules for general litter, a half grade. Similar interactions for the general litter definitions of rules are available, if there is a trace of oil pollution on the beach. Likewise, the occurrence of faeces of non-human origin affects the rules in the input fuzzy set for sewage related debris. At the testing stage, the litter measurements of the field study were compared with the predictions of the fuzzy system, as illustrated in Figure 2.

\section{Conclusions}

Tourist beaches near Antalya region were surveyed in order to develop a standardized fuzzy system approach to be used in litter prediction. The main source of litter on Turkish Mediterranean beaches was determined as "beach users". The main advantage in using fuzzy systems was that they could consider the linguistic definitions/notes of beach users and field study teams during measurements. Therefore, they make effective use of "additional information" such as the knowledge and experience of team members. As a result, additional information inherent in the linguistic comments/refinements and judgment of study teams and beach users could be included in the grading system. Specific issues related to beach characteristics, litter assessment methodology and definition of oil pollution, which could not be included in standard procedures and/or could be easily lost in mathematical expressions/evaluations, were incorporated by using this artificial intelligence system.

\section{References}

1. Balas, C.E., Williams, A.T., Simmons, S.L and A Ergin. A Statistical Riverine Litter Propagation Model, Marine Pollution Bulletin, 42(11), 1169-1176 (2001).

2. EA/NALG, Assessment of Aesthetic Quality of Coastal and Bathing Beaches, Monitoring Protocol and Classification Scheme, Environmental Agency, Bristol, UK (2000).

3. Zadeh, L. A. Fuzzy Logic and the Calculi of Fuzzy Rules, Fuzzy Graphs, and Fuzzy Probabilities, Computer and Mathematics with Applications, Vol. 37, 35 (1999). 